\title{
Supporting Information for: Establishing Meaningful Limits of Detection for Ion-Selective Electrodes and Other Nonlinear Sensors
}

\author{
Peter W. Dillingham, ${ }^{*,+\ddagger}$ Basim S.O. Alsaedi, $\ddagger$ Sergio Granados-Focil,§ Aleksandar Radu,॥ \\ Christina M. McGraw, ${ }^{*, \perp}$ \\ † Department of Mathematics and Statistics, University of Otago, Dunedin 9054, New Zealand \\ ‡ School of Science and Technology, University of New England, Armidale 2351, NSW, Australia \\ $\S$ Sackler Sciences Center, Department of Chemistry, Clark University, Worcester, Massachusetts o1610, United \\ States \\ " Lennard-Jones Laboratories, Birchall Centre, Keele University, Keele, Staffordshire ST5 5 BG, United Kingdom \\ ${ }^{\perp}$ Department of Chemistry, University of Otago, Dunedin 9054, New Zealand
}

\begin{abstract}
SUMMARY: The main section of the paper describes a new definition for limit of detection (LOD) based on false-positive and false-negative rates using the Nikolskii-Eisenman equation (eq 1), along with describing a computational method for estimating LOD for non-linear sensors. It also makes comparisons between the new definition and the current definition of LOD, LOD ${ }_{1969}$, first described in $1969^{1}$ and formalized in $1994^{2}$, linked to the Nernstian approximation for ISEs (eq 4). In the following sections, we provide (1) derivations of LOD and related properties, (2) define variants of LOD when the valences of primary and interfering ions are different for valences $\pm 1, \pm 2$, or when variance is non-constant, (3) provide additional detail on statistical properties of the current LOD definition, (4) derive the limit of discrimination, (5) present expanded simulation results, and (6) present $\mathrm{R}$ code used to analyse the $\mathrm{Pb}^{2+}$ data and create Figure 5.
\end{abstract}

\section{Section 1. Derivation of limit of detection and related properties for the Nikolskii-Eisenman equation based on IUPAC recommendations}

Here we derive five properties related to LOD for the empirical version of the Nikolskii-Eisenman equation (eq 1). ${ }^{1-3}$ Property 1 describes the detection threshold (DT $\alpha$ ) based on false-positive rate $\alpha$, key to the determination of LOD under IUPAC guidelines ${ }^{4}$ and broader recommendations. ${ }^{5-7}$ Property 2 combines the maximum-likelihood estimate for analyte activity with a decision rule, deriving a variant of eq 2 used to derive Properties 3 and 4. Property 3 describes the distribution of the estimated analyte activity given model parameters and actual analyte activity as closely linked to a truncated Log-Normal distribution. In Property 4, the expected value of estimated activity conditional on detection is described. Property 4 can be used to assess bias near DT $\alpha$ or other values of interest due to the truncation that results from the Detected/Not Detected decision rule. Finally, Property 5 derives limit of detection $\left(\operatorname{LOD}_{\alpha, \beta}\right)$ using false-positive rate $\alpha$ and false-negative rate $\beta$. 
Property 1: The probability of detection $\pi_{a}$ at activity $a$ controlling for false-negative rate $\alpha$ is

$$
\pi_{a}=1-\Phi\left[\frac{\left|\beta_{1}\right|}{\sigma} \log \left(\frac{\beta_{2}}{a+\beta_{2}}\right)+z_{\alpha}\right],
$$

where $\Phi$ is the cumulative density function of the Normal distribution, $z_{\alpha}$ is the upper $\alpha$ quantile of a standard normal variate, and $\sigma$ is the standard deviation of the signal noise.

\section{Proof:}

Given the detection threshold at false-negative rate $\alpha, \mathrm{DT}_{\alpha}$, the probability of detection for a given analyte concentration $a, \pi_{a}$, can be formulated as follows:

$$
\pi_{a}=P\left(E>\mathrm{DT}_{\alpha} \mid a\right) \quad(\text { for cations })
$$

or

$$
\pi_{a}=P\left(E<\mathrm{DT}_{\alpha} \mid a\right) \quad \text { (for anions) }
$$

Recognising that eq 1 can be re-written as $E=E^{\circ}+\beta_{1} \log \left(a+\beta_{2}\right)+z \sigma$, where $z$ is a standard normal variate, this is equivalent to

$$
\pi_{a}=P\left(E^{0}+\beta_{1} \log \left(a+\beta_{2}\right)+\epsilon>E^{0}+\beta_{1} \log \left(\beta_{2}\right)+z_{\alpha} \sigma\right) \text { (for cations) }
$$

or

$$
\pi_{a}=P\left(E^{0}+\beta_{1} \log \left(a+\beta_{2}\right)+\epsilon<E^{0}+\beta_{1} \log \left(\beta_{2}\right)-z_{\alpha} \sigma\right) \text { (for anions) }
$$

For both cations and anions, this reduces to

$$
\pi_{a}=P\left(\epsilon>\left|\beta_{1}\right| \log \left(\frac{\beta_{2}}{a+\beta_{2}}\right)+z_{\alpha} \sigma\right) .
$$

Using $\epsilon \sim \mathcal{N}\left(0, \sigma^{2}\right)$ and noting $Z=\epsilon / \sigma$ is a standard normal variate, we then have

$$
\pi_{a}=P\left(Z>\frac{\left|\beta_{1}\right|}{\sigma} \log \left(\frac{\beta_{2}}{a+\beta_{2}}\right)+z_{\alpha}\right),
$$

which is equivalent to

$$
\begin{aligned}
\pi_{a} & =1-P\left(Z<\frac{\left|\beta_{1}\right|}{\sigma} \log \left(\frac{\beta_{2}}{a+\beta_{2}}\right)+z_{\alpha}\right) \\
& =1-\Phi\left[\frac{\left|\beta_{1}\right|}{\sigma} \log \left(\frac{\beta_{2}}{a+\beta_{2}}\right)+z_{\alpha}\right] .
\end{aligned}
$$


Property 2: Given values for $E, E^{0}, \beta_{1}, \beta_{2}$, and $\sigma$, a decision rule where MLE estimates of $\hat{a}<\mathrm{DT}_{\alpha}$ are set to 'Not detected', the likelihood-based point estimates are

$$
\hat{a}= \begin{cases}\left(a+\beta_{2}\right) 10^{\frac{\epsilon}{\beta_{1}}}-\beta_{2}, & \left(a+\beta_{2}\right) 10^{\frac{\epsilon}{\beta_{1}}}-\beta_{2}>\mathrm{DT}_{\alpha} \\ \text { Not detected }, & \text { Otherwise. }\end{cases}
$$

Proof:

First, the log-likelihood for $a$ given the observed emf and model parameters is

$$
l\left(a \mid E, E^{0}, \beta_{1}, \beta_{2}, \sigma\right)=-\frac{1}{2} \ln (2 \pi)-\ln (\sigma)-\frac{1}{2 \sigma^{2}}\left(E-E^{0}-\beta_{1} \log \left(a+\beta_{2}\right)\right)^{2} .
$$

Then, by taking the derivative with respect to $a$ we get the following:

$$
\frac{d l}{d a}=\frac{-1}{\sigma^{2}}\left(E-E^{0}-\beta_{1} \log \left(a+\beta_{2}\right)\right)\left(\frac{-\beta_{1}}{\left(a+\beta_{2}\right)}\right) .
$$

Maximising to find $\hat{a}$ yields eq 2, noting the cancellation of non-zero constants. Substituting for $E$ via eq 1 for positive $\hat{a}$ yields

$$
\hat{a}=\left(a+\beta_{2}\right) 10^{\frac{\epsilon}{\beta_{1}}}-\beta_{2}
$$

For trace elements, the right hand side of the equation (RHS) $<\mathrm{DT}_{\alpha}$ is likely and 'Non-detected' is returned according to the decision rule, leading to eq $\mathrm{S} 2$. We also note that the equation above is equivalent to eq 2, and that $\hat{a}>\mathrm{DT}_{\alpha}$ when $\epsilon \geq \epsilon_{\mathrm{DT}}=\beta_{1} \log \left(\beta_{2} /\left(a+\beta_{2}\right)\right)+z_{\alpha} \sigma$ for cations or $\epsilon \leq \epsilon_{\mathrm{DT}}=\beta_{1} \log \left(\beta_{2} /\left(a+\beta_{2}\right)\right)-z_{\alpha} \sigma$ for anions.

Property 3: Let $\gamma=\hat{a}+\beta_{2}$ for $\hat{a}>\mathrm{DT}_{\alpha}$. The distribution of $\gamma$ given $a$ is $f_{\gamma(a)}=f_{\Theta(a)} / \pi_{a}$, where

$$
f_{\Theta(a)} \sim \log -\text { Normal }\left(\ln \left(a+\beta_{2}\right), \frac{\sigma \ln (10)}{\beta_{1}}\right)
$$

and $\pi_{a}$ is the probability of detection derived in Property 1.

Proof:

First, consider the function $g(\epsilon)=\theta=\left(a+\beta_{2}\right) 10^{\frac{\epsilon}{\beta_{1}}}$. That is, the function $g(\epsilon)$ provides a mapping from the original sampling space of $\epsilon$ (where $\epsilon \sim \mathcal{N}\left(0, \sigma^{2}\right)$ ) to the new sample space of the random variable $\theta$, and the mapping is strictly monotonic and differentiable. Therefore, 


$$
f_{\Theta}=f_{\epsilon}\left(g^{-1}(\theta)\right)\left|\frac{d}{d \theta} g^{-1}(\theta)\right|,
$$

where $g^{-1}(\theta)=\beta_{1} \log \left(\frac{\theta}{a+\beta_{2}}\right)$ and $\left|\frac{d}{d \theta} g^{-1}(\theta)\right|$ is the Jacobian determinant, $\left(\frac{\beta_{1}}{\ln 10} \frac{1}{\theta}\right)$.

This leads to the following form of the distribution of the transformed variable:

$$
f_{\Theta}=\underbrace{\frac{1}{\sigma \sqrt{2 \pi}} \exp \left(\frac{-1}{2 \sigma^{2}}\left(\beta_{1} \log \left(\frac{\theta}{a+\beta_{2}}\right)\right)^{2}\right)}_{f_{\epsilon}\left(g^{-1}(\theta)\right)} \underbrace{\left(\frac{\beta_{1}}{\theta \ln 10}\right)}_{\left|\frac{d}{d \theta} g^{-1}(\theta)\right|} .
$$

Manipulating the logarithm and performing algebra, we get

$$
\begin{gathered}
f_{\Theta}=\frac{\beta_{1}}{\sigma \sqrt{2 \pi} \theta \ln 10} \exp \left(\frac{-1}{2 \sigma^{2}}\left(\beta_{1}\left(\log \theta-\log \left(a+\beta_{2}\right)\right)^{2}\right)\right. \\
=\frac{1}{\theta \sqrt{2 \pi}\left(\frac{\sigma \ln 10}{\beta_{1}}\right)} \exp \left(\frac{-1}{2 \sigma^{2}}\left(\beta_{1}\left(\frac{\ln \theta}{\ln 10}-\frac{\ln \left(a+\beta_{2}\right)}{\ln 10}\right)\right)^{2}\right) \\
=\frac{1}{\theta \sqrt{2 \pi}\left(\frac{\sigma \ln 10}{\beta_{1}}\right)} \exp \left(-\frac{\left(\frac{\beta_{1}}{\ln 10}\right)^{2}}{2 \sigma^{2}}\left(\left(\ln \theta-\ln \left(a+\beta_{2}\right)\right)^{2}\right)\right. \\
=\frac{1}{\theta \sqrt{2 \pi}\left(\frac{\sigma \ln 10}{\beta_{1}}\right)} \exp \left(\frac{-1}{2\left(\frac{\sigma \ln 10}{\beta_{1}}\right)^{2}}\left(\ln \theta-\ln \left(a+\beta_{2}\right)\right)^{2}\right) .
\end{gathered}
$$

Note that the RHS is a log-normal distribution, i.e. $f_{\Theta(a)} \sim \log -\operatorname{Normal}\left(\ln \left(a+\beta_{2}\right), \sigma \ln 10 / \beta_{1}\right)$. Next, we note that $\gamma=\theta$ for $\hat{a}>\mathrm{DT}_{\alpha}$, which occurs with probability $\pi_{a}$ (from Property 1 as opposed to the number $\pi$ ), and thus $f_{\gamma(a)}=f_{\Theta(a)} / \pi_{a}$, completing the derivation of eq S3. That is, $\gamma$ is a truncated Log-Normal variate. 
Property 4: The expected value of $\gamma$ given detection,

$$
E\left(\gamma \mid \gamma>\mathrm{DT}_{\alpha}+\beta_{2}\right)=\frac{\exp \left(\mu+\frac{\left(\sigma^{*}\right)^{2}}{2}\right)}{\pi_{\alpha}} \Phi\left(\frac{\left(\mu+\left(\sigma^{*}\right)^{2}-\ln \left(\mathrm{DT}_{\hat{a}}+\beta_{2}\right)\right)}{\sigma^{*}}\right),
$$

where $\sigma^{*}=\sigma \ln (10) / \beta_{1}$ and, therefore, $E\left(\hat{a} \mid \hat{a}>\mathrm{DT}_{\alpha}\right)=E(\gamma)-\beta_{2}$.

\section{Proof:}

First, note that

$$
E\left(\gamma \mid \gamma>\mathrm{DT}_{\alpha}+\beta_{2}\right)=\frac{1}{\pi_{a}} \int_{\left(\mathrm{DT}_{\alpha}+\beta_{2}\right)}^{\infty} \frac{\gamma}{\gamma \sqrt{2 \pi}\left(\frac{\sigma \ln 10}{\beta_{1}}\right)} \exp \left(\frac{-1}{2\left(\frac{\sigma \ln 10}{\beta_{1}}\right)^{2}}\left(\left(\ln \gamma-\ln \left(a+\beta_{2}\right)\right)^{2}\right) d \gamma .\right.
$$

Next, let $t=\ln (\gamma) \Rightarrow \exp (t)=\gamma$ by taking the derivative of both sides, or $\gamma d t=d \gamma$. The integration limits are adjusted after the substitution $\gamma \rightarrow \infty \Rightarrow \exp (t) \rightarrow \infty$, and $\gamma=\mathrm{DT}_{\alpha}+\beta_{2} \Rightarrow t=\ln \left(\mathrm{DT}_{\alpha}+\beta_{2}\right)$. Substituting $\mu=\ln \left(a+\beta_{2}\right)$ and $\sigma^{*}=\sigma \ln (10) / \beta_{1}$, the integral can be rewritten as

$$
\begin{aligned}
& =\frac{1}{\pi_{a}} \int_{\ln \left(\mathrm{DT}_{\alpha}+\beta_{2}\right)}^{\infty} \frac{1}{\sqrt{2 \pi}\left(\sigma^{*}\right)} \exp \left(\frac{-1}{2\left(\sigma^{*}\right)^{2}}(t-\mu)^{2}\right) \exp (t) d t \\
& =\frac{\exp \left(\mu+\frac{\left(\sigma^{*}\right)^{2}}{2}\right)}{\pi_{a}} \int_{\frac{1}{\sigma^{*}\left(\ln \left(\mathrm{DT}_{\alpha}+\beta_{2}\right)-\mu-\sigma^{* 2}\right)}}^{\infty} \frac{\sigma^{*}}{\sqrt{2 \pi}\left(\sigma^{*}\right)} \exp \left(\frac{-1}{2} s^{2}\right) d s .
\end{aligned}
$$

By completing the square, $t-\frac{1}{2\left(\sigma^{*}\right)^{2}}(t-\mu)^{2}=-\frac{1}{2\left(\sigma^{*}\right)^{2}}\left(t-\left(\mu+\sigma^{* 2}\right)\right)^{2}+\mu+\frac{\sigma^{* 2}}{2}$, the integral then becomes

$$
E\left(\gamma \mid \gamma>\mathrm{DT}_{\alpha}+\beta_{2}\right)=\frac{\exp \left(\mu+\frac{\left(\sigma^{*}\right)^{2}}{2}\right)}{\pi_{a}} \int_{\ln \left(\mathrm{DT}_{\alpha}+\beta_{2}\right)}^{\infty} \frac{1}{\sqrt{2 \pi}\left(\sigma^{*}\right)} \exp \left(\frac{-1}{2\left(\sigma^{*}\right)^{2}}\left(t-\left(\mu+\sigma^{* 2}\right)\right)^{2}\right) d t
$$

Letting $s=\left(t-\left(\mu+\sigma^{* 2}\right)\right) / \sigma^{*}$, we have $\sigma^{*} s+\left(\mu+\sigma^{* 2}\right)=t \Rightarrow s=\left(t-\mu-\sigma^{* 2}\right) / \sigma^{*}$. By differentiating, we get $\sigma^{*} d s=d t$. The integration limits are adjusted after the substitution, $t \rightarrow \infty \Rightarrow s \rightarrow \infty, t=\ln \left(\mathrm{DT}_{\alpha}+\beta_{2}\right) \Rightarrow s=\left(\ln \left(\mathrm{DT}_{\alpha}+\beta_{2}\right)-\mu-\sigma^{* 2}\right) / \sigma^{*}$. Therefore, 


$$
\begin{aligned}
& E\left(\gamma \mid \gamma>\mathrm{DT}_{\alpha}+\beta_{2}\right)=\frac{\exp \left(\mu+\left(\sigma^{*}\right)^{2} / 2\right)}{\pi_{a}} \int_{\left(\ln \left(\mathrm{DT}_{\alpha}+\beta_{2}\right)-\mu-\sigma^{* 2}\right) / \sigma^{*}}^{\infty} \frac{\sigma^{*}}{\sqrt{2 \pi}\left(\sigma^{*}\right)} \exp \left(\frac{-1}{2} s^{2}\right) d s \\
& =\frac{\exp \left(\mu+\left(\sigma^{*}\right)^{2} / 2\right)}{\pi_{a}}[\underbrace{\int_{-\infty}^{\infty} \frac{1}{\sqrt{2 \pi}} \exp \left(\frac{-1}{2} s^{2}\right) d s}_{1}-\underbrace{\underbrace{\left(\ln \left(\mathrm{DT}_{\alpha}+\beta_{2}\right)-\mu-\sigma^{* 2}\right) / \sigma^{*}}_{-\infty} \int_{-\infty}^{1} \exp \left(\frac{-1}{2} s^{2}\right) d s}_{\text {CDF of the standard normal }}] .
\end{aligned}
$$

Since the standard normal distribution is symmetric,

$$
1-\Phi\left(\frac{1}{\sigma^{*}}\left(\ln \left(\mathrm{DT}_{\alpha}+\beta_{2}\right)-\mu-\sigma^{* 2}\right)\right)=\Phi\left(\frac{\mu+\sigma^{* 2}-\ln \left(\mathrm{DT}_{\alpha}+\beta_{2}\right)}{\sigma^{*}}\right)
$$

and therefore eq S4 follows,

$$
E\left(\gamma \mid \gamma>\mathrm{DT}_{\alpha}+\beta_{2}\right)=\frac{\exp \left(\mu+\left(\sigma^{*}\right)^{2} / 2\right)}{\pi_{a}} \Phi\left(\frac{\left(\mu+\left(\sigma^{*}\right)^{2}-\ln \left(\mathrm{DT}_{\alpha}+\beta_{2}\right)\right)}{\sigma^{*}}\right)
$$

The natural consequence is the that the expected value of estimated activity given detection is

$$
E\left(\hat{a} \mid \hat{a}>\mathrm{DT}_{\alpha}\right)=\frac{\exp \left(\mu+\left(\sigma^{*}\right)^{2} / 2\right)}{\pi_{a}} \Phi\left(\frac{\left(\mu+\left(\sigma^{*}\right)^{2}-\ln \left(\mathrm{DT}_{\alpha}+\beta_{2}\right)\right)}{\sigma^{*}}\right)-\beta_{2} .
$$

Property 5: The limit of detection with false-positive rate $\alpha$ and false-negative rate $\beta$, on the activity scale, is

$$
\operatorname{LOD}_{\alpha, \beta}=\beta_{2}\left(10^{\left(z_{\alpha}+z_{\beta}\right) \sigma|| \beta_{1} \mid}-1\right)
$$

Proof:

The detection threshold with false-positive rate $\alpha$ (eq 7) is

$$
\mathrm{DT}_{\alpha}=E^{\circ}+\beta_{1} \log \beta_{2}+\operatorname{sign}(v) z_{\alpha} \sigma
$$

Limit of detection, on the other hand, is the value of $a$ that exceeds (positively for cations or negatively for anions) the detection threshold with probability $1-\beta$. On the activity scale, this means that $\operatorname{LOD}_{\alpha, \beta}$ occurs when the random variate is below (cations) or above (anions) the upper $\beta$ quantile of its distribution, or

$$
E^{\circ}+\beta_{1} \log \left(\mathrm{LOD}_{\alpha, \beta}+\beta_{2}\right)-\operatorname{sign}(v) z_{\beta} \sigma=\mathrm{DT}_{\alpha}
$$

where $z \beta$ is the upper $\beta$ quantile of the standard normal distribution. Combining with eq 5 and solving for $\mathrm{LOD}_{\alpha, \beta}$ yields the desired result. 


\section{Section 2. LOD when the valence of the interfering ion is different from the primary ion}

When valences of interfering ions are different from the primary ion, eq 1 approximates the true response, which includes nonlinear terms within the logarithm. ${ }^{8}$ A parallel approach to deriving LOD can be taken that incorporates the nonlinear terms (Properties 6 and 7 below). However, the Nikolskii-Eisenman equation with empirical parameter estimates provides a reasonable basis for calculation for many realworld settings, i.e. the bias is small relative to overall uncertainty when considering predictions. The alternate equations are preferable for low-noise sensors where small functional differences can be detected or when considering theoretical behaviour between Nikolskii coefficients, interfering ion activity, and LOD.

Property 6: The limit of detection for a primary ion of valence $v= \pm 1$ and interfering ion of valence \pm 2 (but the same sign as the primary ion) is

$$
\operatorname{LOD}_{\alpha, \beta}=\beta_{2} 10^{\left(z_{\alpha}+z_{\beta}\right) \sigma\left|\beta_{1}\right|}-\beta_{2} /\left(10^{\left(z_{\alpha}+z_{\beta}\right) \sigma\left|\beta_{1}\right|}\right) .
$$

Proof: The emf response can be described by $E=E^{\circ}+\beta_{1} \log \left(a / 2+\left(a^{2}+4 \beta_{2}^{2}\right)^{1 / 2} / 2\right)+\varepsilon^{8}$, with theoretical values for $\beta_{1}=\operatorname{sign}(v) 2.303 R T F^{-1}$ and $\beta_{2}=k_{I J}^{p o t} \sqrt{a_{J}}$, where $a_{J}$ is the activity of the interfering ion and $k_{I J}^{p o t}$ is the Nikolskii coefficient. Following similar calculations as above, the detection threshold is $\mathrm{DT}_{\alpha}=E^{\circ}+\beta_{1} \log \beta_{2}+\operatorname{sign}(v) z_{\alpha} \sigma$. Combining this with

$$
E^{\circ}+\beta_{1} \log \left(\operatorname{LOD}_{\alpha, \beta} / 2+\left(\operatorname{LOD}_{\alpha, \beta}^{2}+4 \beta_{2}^{2}\right)^{1 / 2} / 2\right)-\operatorname{sign}(v) z_{\beta} \sigma=\mathrm{DT}_{\alpha}
$$

and performing algebra yields the desired result.

Property 7: The limit of detection for a primary ion of valence $v= \pm 2$ and interfering ion of valence \pm 1 (but the same sign as the primary ion) is

$$
\operatorname{LOD}_{\alpha, \beta}=\beta_{2} 10^{\left(z_{\alpha}+z_{\beta}\right) \sigma|| \beta_{1} \mid}\left(10^{\left(z_{\alpha}+z_{\beta}\right) \sigma|| \beta_{1} \mid}-1\right) .
$$

Proof: The emf response can be described by $E=E^{\circ}+\beta_{1} \log \left(\left(a+\beta_{2} / 4\right)^{1 / 2}+\left(\beta_{2} / 4\right)^{1 / 2}\right)+\varepsilon^{8}$, with theoretical values for $\beta_{1}=\operatorname{sign}(v) 2.303 R T F^{-1}$ and $\beta_{2}=k_{I J}^{p o t} a_{J}^{2}$. The detection threshold is described by $\mathrm{DT}_{\alpha}=E^{\circ}+0.5 \beta_{1} \log \beta_{2}+\operatorname{sign}(v) z_{\alpha} \sigma$. Combining this with

$$
E^{\circ}+\beta_{1} \log \left(\left(\operatorname{LOD}_{\alpha, \beta}+\beta_{2} / 4\right)^{1 / 2}+\left(\beta_{2} / 4\right)^{1 / 2}\right)-\operatorname{sign}(v) z_{\beta} \sigma=\mathrm{DT}_{\alpha}
$$

and performing algebra yields the desired result. 


\section{Section 3. LOD when variance is not constant}

In some cases, the assumption of constant variance in eq 1 may not hold, e.g. the noise for a blank may be different than the noise for non-blanks. This may manifest in different ways, including all values other than a blank have a constant variance or variance changing in a more complex manner.

Property 8: The detection threshold and limit of detection where the assumption of constant variance fails are

$$
\mathrm{DT}_{\alpha}=E^{0}+\beta_{1} \log \left(\beta_{2}\right)+\operatorname{sign}(v) z_{\alpha} \sigma_{\text {blank }},
$$

and

$$
\operatorname{LOD}_{\alpha, \beta}=\beta_{2}\left(10^{\left(z_{\alpha} \sigma_{\text {blank }}+z_{\beta} \sigma_{\mathrm{LOD}}\right) /\left|\beta_{1}\right|}-1\right),
$$

where $\sigma_{\text {blank }}$ is the standard deviation of a blank and $\sigma_{\text {LOD }}$ is the standard deviation at the LOD. Other parameters are defined as before.

Proof: The proofs follow the same logic as establishing DT $\alpha$ and $\operatorname{LOD}_{\alpha, \beta}$ under eq 1 , but removing the constant variance assumption.

\section{Practical considerations.}

Implementation of eq S8 may be difficult as it requires at least some knowledge of the relationship between activity $a$ and the corresponding noise $\left(\sigma_{a}\right)$, e.g. $\sigma_{a}=f\left(\sigma_{\text {blank }}, a\right)$ for some function $f$. This may require extra data collection (e.g. to establish $\sigma_{\text {blank }}$ as well as noise levels at other values of $a$ ), a modification of the Bayesian model (to allow for varying levels of noise), or the use of a numerical optimizer unless $f$ is of a form that allows an algebraic solution (e.g. $\sigma_{\text {LOD }} \approx 2 \sigma_{\text {blank }}$ or $\sigma_{a}$ is constant). However, these are all tractable problems with straightforward solutions.

The benefit of allowing for non-constant variance for estimating LOD and other parameters of interest would depend on how much the noise levels change across the observed data, as well as other factors such as the noise level of a blank, ion charge, and the location of calibration data on the activity scale. Simulations suggest that $\sigma_{\mathrm{LOD}} \leq 2 \sigma_{\text {blank }}$ yields bias $<0.3$ in $\log \operatorname{LOD} \alpha, \beta$ for many real-world settings $(10$ or fewer calibration points, maximum noise $<5$ times the noise of a blank) and that this bias reduces for less noisy sensors. 


\section{Section 4. Statistical properties of the current LOD definition}

The current IUPAC definition of LOD for ISEs $\left(\operatorname{LOD}_{1969)}\right)$ lies at the intersection of the blank and Nernstian responses of the Nikolskii-Eisenman Equation. ${ }^{9}$ On the activity scale, $\operatorname{LOD}_{1969}=\beta_{2}$ (eq 8).

Often, this leads to bias in estimation, e.g. if the Nernstian approximation is used near LOD 1969 (Figure S1). In the context of general IUPAC recommendations for LOD, this leads to flaws including potential estimation bias near LOD, an undefined detection threshold, and false-positive and -negative rates with poor behaviour.

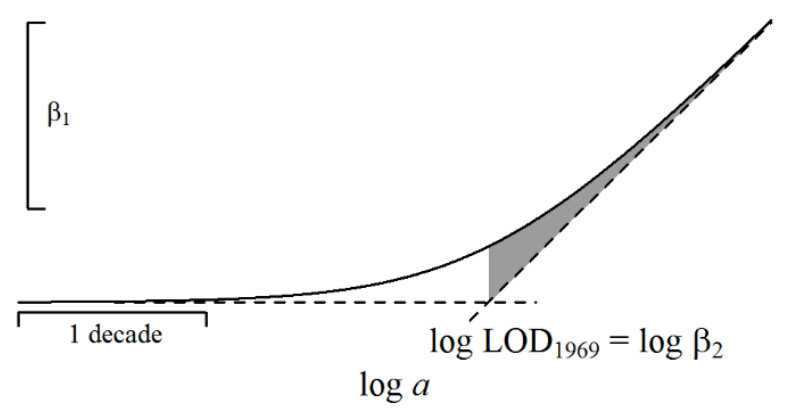

Figure S1. The current definition of LOD for ISEs $\left(\operatorname{LOD}_{1969}\right)$ lies at the intersection of the blank and Nernstian responses of the Nikolskii-Eisenman Equation. If the Nernstian approximation is used to estimate activity near $\mathrm{LOD}_{1969}$, substantial bias occurs for approximately one order of magnitude (shaded region).

The current definition of LOD for ISEs does not distinguish between DT and LOD, and the lack of clarity around detection carries through to uncertainty as to the calculation of detection probability. We consider two possible approaches to translating LOD 1969 to a DT. The first approach (a) treats any observation with estimated activity greater than $\mathrm{LOD}_{1969}$ using the Nernst approximation as detected, while the second approach (b) uses any estimated activity from the Nikolskii-Eisenman Equation $>\operatorname{LOD}_{1969}$ as a detection rule. If using the Nernstian approximation near $\mathrm{LOD}_{1969}$ to estimate activity, substantial bias occurs for approximately one order of magnitude (Figure S1). On the emf scale, these two approaches translate to emf detection thresholds of (a) $E^{\circ}+\beta_{1} \log \beta_{2}$ and (b) $E^{\circ}+\beta_{1} \log 2 \beta_{2}$. For any activity $a$, the probability of detection $\left(\pi_{1969}\right)$ is derived in a manner analogous to Property 1 , yielding:

$$
\pi_{1969}=1-\Phi\left[\left|\beta_{1}\right| \log \left(\beta_{2} /\left(a+\beta_{2}\right)\right) / \sigma\right]
$$

or

$$
\pi_{1969}=1-\Phi\left[\left|\beta_{1}\right| \log \left(2 \beta_{2} /\left(a+\beta_{2}\right)\right) / \sigma\right] .
$$

for the two approaches, where $\Phi$ is the cumulative density function for a standard normal distribution. There are, of course, other ways that a DT could be created from the current IUPAC definition; we suggest detection functions for alternatives would likely lie between Equations S9a and S9b. 
Using IUPAC recommendations for LOD, the detection probability at $a=0$ (i.e. a blank) should equal $\alpha$, while the detection probability at $a=\mathrm{LOD}$ should equal $1-\beta$. However, at $a=0, \pi_{1969}=0.5$ (eq S9a) or remains a function of $\beta_{1}$ and $\sigma$ (eq S9b), decreasing for low valence ions and less noisy sensors. Under approach (a), any positive random noise leads to $E>E^{\circ}+\beta_{1} \log \beta_{2}$ for cations and any negative random error leads to $E<E^{\circ}+\beta_{1} \log \beta_{2}$ for anions. That is, this approach leads to a false detection rate of $50 \%$, ten times the recommended standard rate of $\alpha=0.05$. Under approach (b), for some ISEs the falsenegative rate may be essentially 0 (e.g. valence \pm 1 with $\sigma<5 \mathrm{mV}$ ), while others may have more than twice the nominal false-positive rate of 0.05 (e.g. valence \pm 3 with $\sigma>5 \mathrm{mV}$ ).

At $a=\mathrm{LOD}_{1969}$, a similar pattern occurs, but in reverse. For approach (a), $\pi_{1969}$ varies depending on ISE model parameters but does not equal the targeted $1-\beta$ : the detection probability increases for low valence ions and less noisy sensors ( $\pi_{1969}$ is often close to 1 ) and decreases for high valence ions and noisier sensors $\left(\pi_{1969}<0.8\right.$ would not be unusual). For approach $(b), \pi_{1969}=0.5$ for all ISEs, i.e. the falsenegative rate would be ten times the recommended standard rate of $\beta=0.05$.

\section{Section 5. Limit of discrimination}

Closely related to limit of detection is the limit of discrimination. Rather than comparing to a blank, of interest is discrimination from a non-zero value $R$, e.g. a regulatory limit. In this case, detection occurs at $\mathrm{DT}_{R, \alpha}=E^{\circ}+\beta_{1} \log \left(R+\beta_{2}\right)+\operatorname{sign}(v) z_{\alpha} \sigma$. Proceeding as in the derivation for LOD (Equation S5) yields the limit of discrimation,

$$
\operatorname{LOD}_{R, \alpha, \beta}=\left(R+\beta_{2}\right) 10^{\left(z_{\alpha}+z_{\beta}\right) \sigma\left|\beta_{1}\right|}-\beta_{2} .
$$

\section{Section 6. Expanded simulation results}

Three simulations were performed to assess the performance of the conditional analytic (CA) method, with key results given in the main text. Here, we provide additional detail on each. For the first simulation based on a small sample size $(n=7)$, bias was small but non-zero (Figure S2a). The Bayesian model used to estimate model parameters and LOD employed broad priors, particularly for $\sigma$, to accommodate the range of values used in the simulations. This leads to positive bias in $\hat{\sigma}$ for small $\sigma$ and negative bias for large $\sigma$, translating into bias in LOD (Figure S2a). In settings where more prior information on $\sigma$ was available, reduced bias in $\sigma$ and hence LOD would result, also improving coverage. However, even with the broad prior for $\sigma$, bias is sufficiently low and coverage near enough to nominal rates for most applications. 

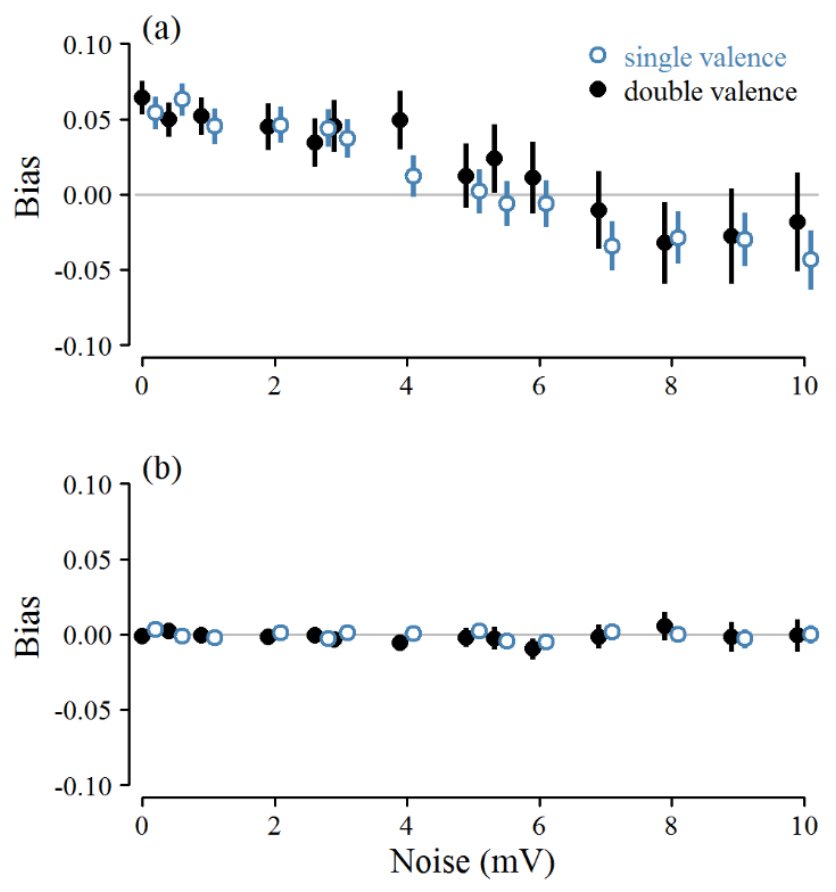

Figure S2. Estimated bias of $\log \operatorname{LOD}_{\alpha, \beta}$ with (a) $n=7$ and (b) $n=601$ calibration points. Vertical lines represent $95 \%$ confidence intervals from (a) 1,000 and (b) 100 simulations. For plotting clarity, noise $(\sigma)$ was shifted 0.1 units left (single valence) or right (double valence) from actual values.

Coverage - the rate at which the nominal 95\% credible intervals contain the true LOD - was near 95\%, ranging from 94-97\%, with slight undercoverage for small values of $\sigma$ and slight overcoverage for large values (Figure S3); credible intervals are based on a beta-binomial model with a non-informative prior distribution for coverage of beta $(0.0001,0.0001)$.

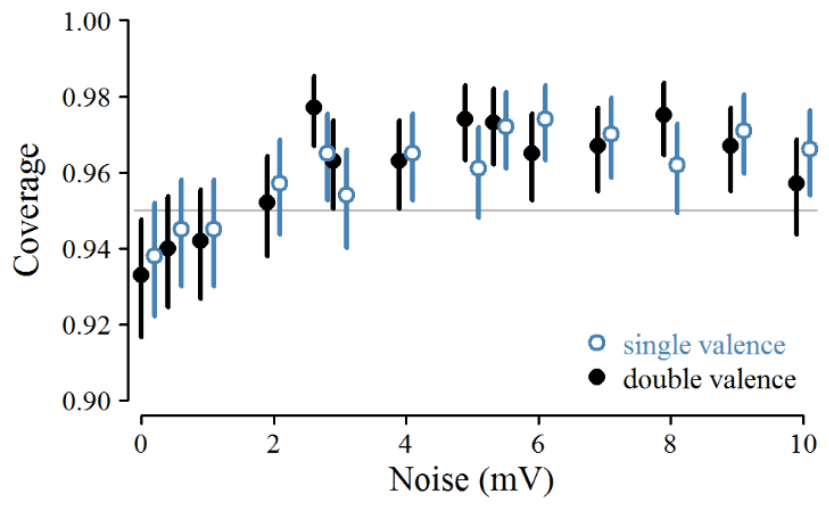

Figure S3. Estimated coverage of $\log \operatorname{LOD}_{\alpha, \beta}$ with $n=7$. Vertical lines represent $95 \%$ credible intervals from 1,000 simulations using a beta-binomial model. For plotting clarity, noise $(\sigma)$ was shifted 0.1 units left (single valence) or right (double valence) from actual values. 
Secondary simulations were performed to assess large sample performance. The first of these secondary simulations measured RMSE, bias, and coverage of $95 \%$ credible intervals for $\log \operatorname{LOD}_{\alpha, \beta}$ for each of the 28 combinations of valence and noise described in the main text. For each combination, $R=100$ simulated datasets were generated using $n=601$ calibration points, spaced at 0.01 intervals on the log activity scale. These results were combined to estimate RMSE, bias, and coverage. The second simulation assessed the same metrics as the first, but as a function of sample size $(n=7,13,25,61,121,241$, or 601 equally spaced calibration points) with $R=1,000$ simulated datasets for the single scenario where $\beta_{1}=$ $29.6 \mathrm{mV} /$ decade and $\sigma=3$.

For the second, larger sample size simulations, model behaviour was consistent with theoretical expectations: $\log \mathrm{LOD}_{\alpha, \beta}$ showed negligible bias (Figure $\mathrm{S} 2 \mathrm{~b}$ ), coverage showed no pattern versus $\sigma$ and was at or near the nominal rate (overall coverage 0.9461; s.e. 0.0045), and it substantially outperformed $\log$ LOD $_{1969}$ nearly everywhere on an RMSE basis (Figure S4). For RMSE, the only exception occurred where $\mathrm{LOD}_{\alpha, \beta}=\mathrm{LOD}_{1969}$ and the simpler estimator must outperform the more complex estimator due to its inherent greater variance; even here, the difference was small ( $9 \%$ increase in RMSE for valence 1 , $19 \%$ increase for valence 2). Finally, the simulation assessing performance as a function of sample size found coverage rates from 0.95 to 0.97 (s.e. 0.007 ), close to the nominal rate of $95 \%$. The slight overcoverage was observed for $n=7$ and consistent with the results shown in Figure S3, with all other values near $95 \%$. As expected, RMSE decreased approximately with $\sqrt{n}$.
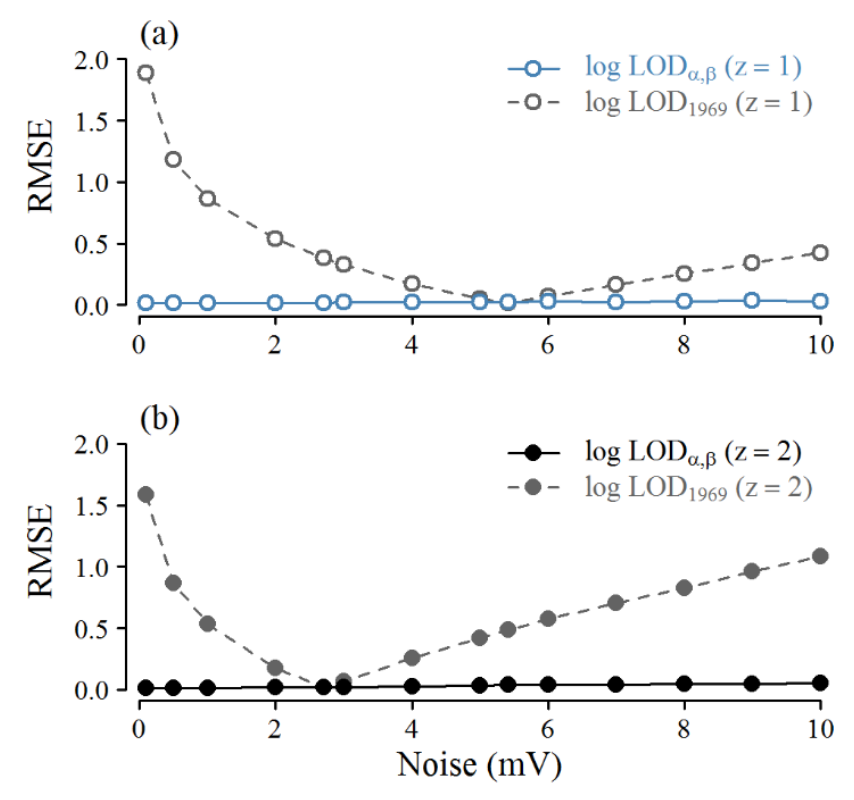

Figure S4. A comparison of the performance of two estimators for $\operatorname{LOD}_{\alpha, \beta}$ shows that $\log \operatorname{LOD}_{\alpha, \beta}$ outperforms $\log \operatorname{LOD}_{\alpha, \beta}$ on an RMSE basis nearly everywhere in a large sample setting $(n=601)$ for single valence (a) and double valence (b) ions. Each point is based on the average results from 100 simulated datasets; error bars are smaller than the plotting symbol. 


\section{Section 7. $\mathbf{R}$ code used to analyse $\mathbf{P b}^{2+}$ data and create Figure 4.}

Below, we present $\mathrm{R}$ code used to analyse the $\mathrm{Pb}^{2+}$ data and create Figure 4. This is intended to demonstrate advanced features of ISEtools, ${ }^{3,10}$ where model output is saved and used to create a bespoke figure. Users may save figures in other formats easily, e.g. using tiff, pdf, or postscript commands.

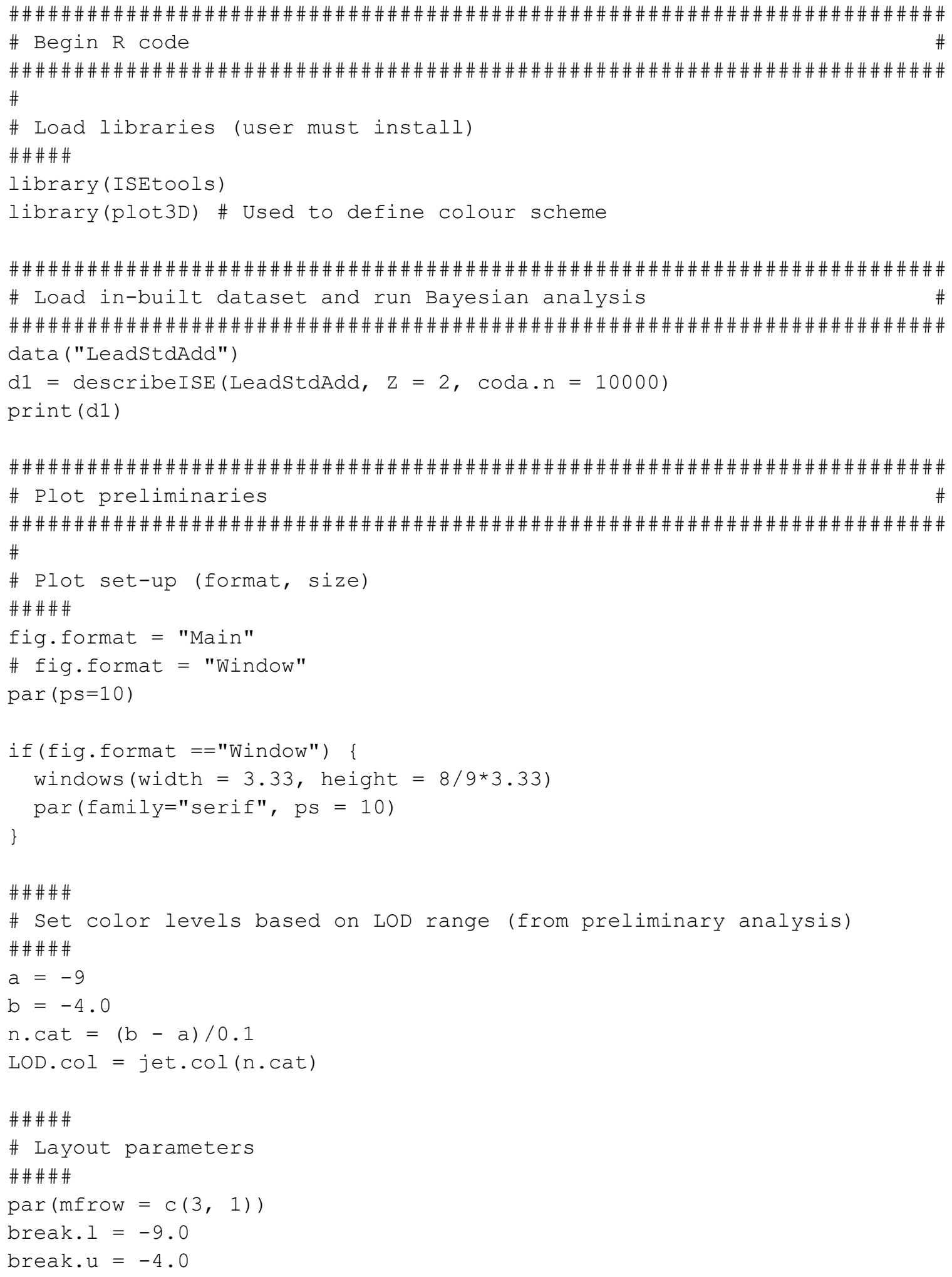




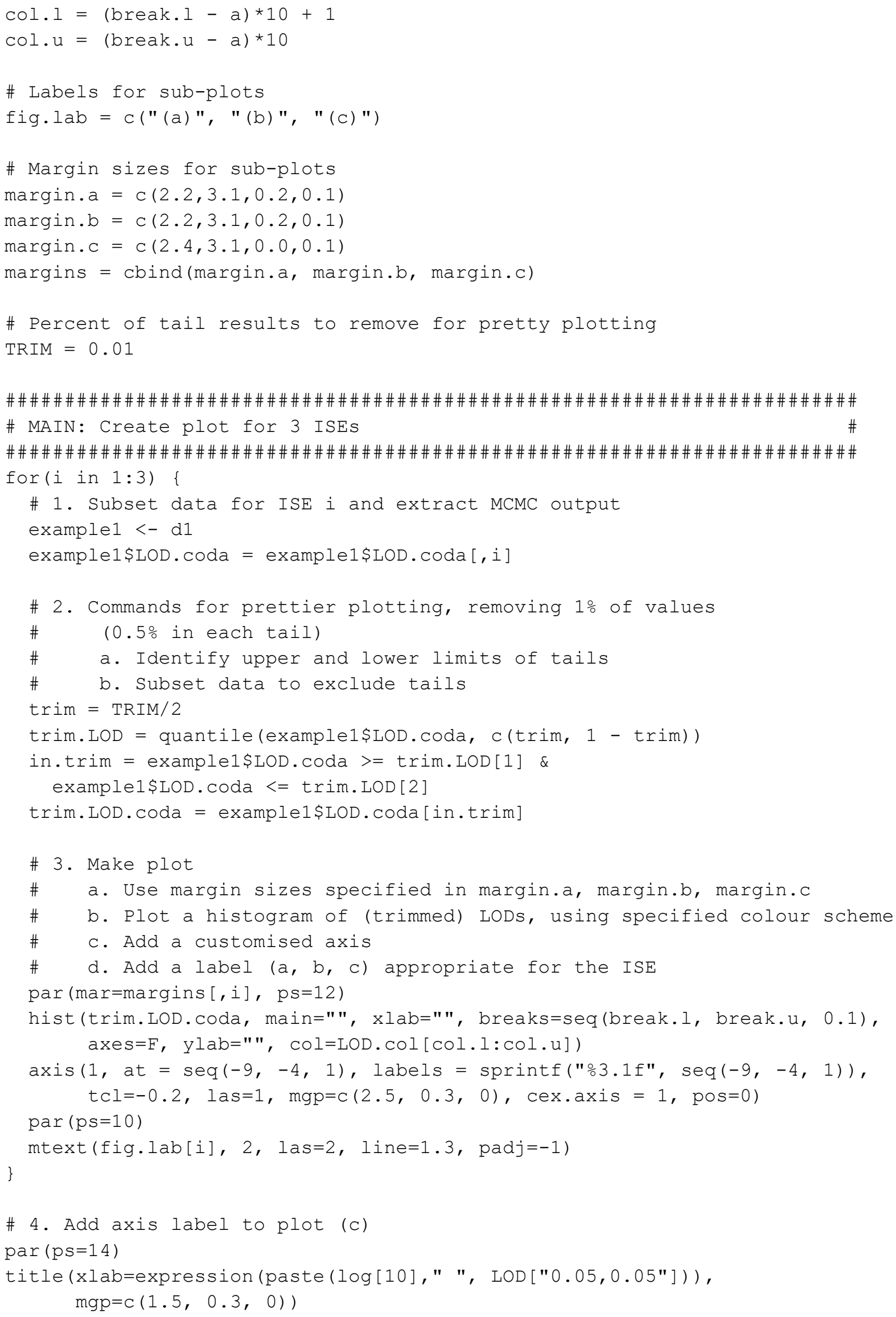




\section{References}

1. Eisenman, G.; Rudin, D. O.; Casby, J. U., Glass Electrode for Measuring Sodium Ion. Science 1957, 126 (3278), 831-834.

2. Dillingham, P. W.; Radu, T.; Diamond, D.; Radu, A.; McGraw, C. M., Bayesian Methods for Ion Selective Electrodes. Electroanal 2012, 24 (2), 316-324.

3. Dillingham, P. W.; Alsaedi, B. S. O.; McGraw, C. M., Characterising uncertainty in instrumental limits of detection when sensor response is non-linear. Ieee Sensor 2017, 94-96.

4. Currie, L. A., Nomenclature in Evaluation of Analytical Methods Including Detection and Quantification Capabilities (Iupac Recommendations 1995). Pure Appl Chem 1995, 67 (10), 1699-1723. 5. Montville, D.; Voigtman, E., Statistical properties of limit of detection test statistics. Talanta 2003, 59 (3), 461-476.

6. Eksperiandova, L. P.; Belikov, K. N.; Khimchenko, S. V.; Blank, T. A., Once Again About Determination and Detection Limits. J Anal Chem+ 2010, 65 (3), 223-228.

7. Desimoni, E.; Brunetti, B., Presenting Analytical Performances of Electrochemical Sensors. Some Suggestions. Electroanal 2013, 25 (7), 1645-1651.

8. Bakker, E.; Meruva, R. K.; Pretsch, E.; Meyerhoff, M. E., Selectivity of polymer membranebased ion-selective electrodes: Self-consistent model describing the potentiometric response in mixed ion solutions of different charge. Analytical Chemistry 1994, 66 (19), 3021-3030.

9. Buck, R. P.; Lindner, E., Recommendations for nomenclature of ion-selective electrodes (IUPAC Recommendations 1994). Pure Appl Chem 1994, 66 (12), 2527-2536.

10. Dillingham, P. W.; Alsaedi, B. S. O.; Radu, A.; McGraw, C. M., Semi-Automated Data Analysis for Ion-Selective Electrodes and Arrays Using the R Package ISEtools. Sensors 2019, 19 (20), 4544. 